\title{
Financial Implications of Pancreas Transplant Complications: A Business Case for Quality Improvement
}

\author{
J. A. Cohn, M. J. Englesbe, Y. M. Ads, J. L. \\ Paruch, S. J. Pelletier, T. H. Welling, C. J. \\ Sonnenday, J. C. Magee, J. D. Punch, D. A. \\ Campbell, Jr. and R. S. Sung
}

Department of Surgery, Division of Transplantation, University of Michigan Health System, Ann Arbor, MI

* Corresponding author: Michael J. Englesbe,

englesbe@med.umich.edu

We quantified the financial implications of surgical complications following pancreas transplantation. We reviewed medical and financial records of 49 pancreas transplant recipients at the University of Michigan Health System (UMHS) between 1/6/2002 and $11 / 22 / 2004$. The association of donor, transplant recipient and financial variables was assessed. The median costs to UMHS of procedures and follow-up were $\$ 92,917$ for recipients without surgical complications versus $\$ \mathbf{1 0 8 , 4 3 1}$ when a surgical complication occurred, a difference of $\$ 15,514(p=0.03)$. Median reimbursement by the payer was $\$ 17,363$ higher in patients with a surgical complication $(p=0.001)$. Similar trends (higher insurer costs) were noted when stratifying by payer (public and private) and specific procedure (SPK and PAK). All parties (patient, physician, payer and medical center) should benefit from quality improvement, with payers having a financial interest in pancreas transplant surgical quality initiatives.

Received 24 July 2006 revised 30 January 2007; accepted 5 February 2007.

The National Surgery Quality Improvement Program (NSQIP) initiated within the VA Hospital System was associated with a $27 \%$ reduction in mortality and a $45 \%$ reduction in morbidity in surgical patients over a 10-year interval (1). In 1999, the American College of Surgeons began implementation of a private-sector NSQIP at academic medical centers in general, vascular and urologic surgery. We recently suggested the implementation of a national transplant surgery quality improvement program (2). We propose that transplant surgeons and payers are uniquely qualified to lead the effort to improve the quality of transplant surgery.
Unfortunately, well-structured quality improvement efforts are expensive, requiring staff and time to collect and analyze data, adding to the burden of mandatory clinical data reporting that already exists. Transplant teams already submit data to the Organ Procurement Transplant Network (OPTN) as required by the Department of Health and Human Services. Medical centers also have substantial data reporting requirements mandated by the Joint Commission on Accreditation of Hospital Organizations (JCAHO) and Center for Medicare Services (CMS). Though costly and time consuming, the transplant community and transplant recipients have benefited from the thoughtful analyses generated from this data.

We believe that a transplant surgical quality improvement program, led by the transplant community, would be an excellent investment. In addition to their harmful effects on the patient, poor surgical outcomes are associated with significantly increased costs to both the medical center and payer. Little data are available on the finances of pancreas transplantation. Gruessner et al. evaluated the risk factors associated with increased hospital charges for pancreas transplant and stated a need for an analysis of hospital costs (3). We have attempted to build on this effort by adding hospital costs and total insurer reimbursement to the analysis. In this study, we compare the costs of care for pancreas transplant recipients who suffer a surgical complication to those without a surgical complication and determine which parties have the largest financial stake in preventing surgical complications. The results suggest that surgical quality improvement initiatives may be a worthwhile financial investment for payers.

\section{Methods}

\begin{abstract}
Clinical data
Following Institutional Review Board approval, the electronic records for all 58 adult pancreas transplant recipients operated on between 1/1/02 and $11 / 15 / 04$ at the University of Michigan Health System (UMHS) were retrospectively evaluated. Data regarding donor, transplant, and recipient characteristics as well as graft and patient outcomes were obtained from both a prospectively collected database and review of the electronic medical record. Nine patients without corresponding financial data were excluded from the analysis, leaving 49 patients who constituted the study group. All pancreas transplants were performed utilizing systemic venous drainage and enteric exocrine drainage. The immunosuppressive regimen consisted
\end{abstract}


Cost of Pancreatic Transplant Complications

Table 1: Background data of patients with no surgical complication vs. those with a surgical complication (data reported as mean \pm SD)

\begin{tabular}{|c|c|c|c|c|}
\hline & $\begin{array}{l}\text { No surgical } \\
\text { complication } \\
(n=30)\end{array}$ & $\begin{array}{l}\text { Surgical } \\
\text { complication } \\
(\mathrm{n}=19)\end{array}$ & $\begin{array}{l}\text { Mean } \\
\text { difference }\end{array}$ & p-Value \\
\hline Recipient age (years) & $38.7 \pm 5.9$ & $38.1 \pm 5.7$ & 0.6 & 0.74 \\
\hline Recipient sex (male) & $16(53.3 \%)$ & $14(73.7 \%)$ & $\mathrm{n} / \mathrm{a}$ & 0.15 \\
\hline Donor age (years) & $26.2 \pm 10.8$ & $23.2 \pm 9.5$ & 3.0 & 0.34 \\
\hline Cold ischemia time (h) & $7.8 \pm 2.3$ & $10.7 \pm 2.5$ & -2.9 & 0.002 \\
\hline Length of stay (days) & $5.8 \pm 1.9$ & $11.5 \pm 10.5$ & -5.7 & 0.005 \\
\hline Readmissions first 6 months & $0.93 \pm 1.2$ & $1.47 \pm 1.2$ & -0.54 & 0.12 \\
\hline
\end{tabular}

of induction therapy with either basiliximab or Thymoglobulin, and triple drug maintenance therapy with Tacrolimus, Mycophenolate mofetil and steroids.

\section{Definition of a surgical complication}

For the purposes of this study, surgical complications were divided into several general categories: bleeding, infection, thrombosis and other complications. 'Other' complications included those not covered by the other three categories and included: delayed kidney graft function (for SPK transplant), acute tubular necrosis (for PTA or PAK transplants), prolonged intubation, prolonged ileus, internal hernia, small bowel obstruction and leaking wound. There were four patients who developed postsurgical urinary tract infections, three who had graft rejection, and two who had other nonsurgical complications. These patients were characterized along as having 'no surgical complication'. Analysis was limited to complications diagnosed within the first six months following transplant. Because pancreas transplant alone procedures (PTA) were uncommon, these were categorized along with pancreas after kidney procedures (PAK) as solitary pancreas transplants. These were compared with simultaneous pancreas-kidney transplants for complication rates and finances.

\section{Financial data}

Inpatient and outpatient financial data were obtained on combined physician procedural and nonprocedural reimbursement, inpatient and outpatient hospital total costs (direct and indirect costs), organ acquisition costs and reimbursements (based on estimates derived from previous reimbursement history) and hospital reimbursements on each patient from the internal costaccounting database at the University of Michigan. All costs for pharmaceuticals, including prolonged inpatient and outpatient intravenous antibiotics for patients, were incorporated into the financial data. Financial data were collected from the day of transplant (including the transplant operation) to 6 months posttransplant. Costs of care outside of UMHS were not obtained. The TSI system (Transitions Systems Inc.) was used to identify total hospital costs (direct and indirect and including organ acquisition costs) and reimbursements. Reimbursements to the medical center were calculated based on a modeled revenue for reimbursements (constantly updated average for a payer based on hospital charges). In TSI, we adjust the Medicare organ acquisition add-on payment based on the final Medicare Cost Report. The TSI system tracks the use of all resources and assigns estimates of cost based on direct acquisition costs for supplies and time-and-motion studies for labor costs. This method of activity-based cost accounting is widely believed to be the most accurate method of estimating the true economic cost of an episode of care (4). Hospital margins were calculated by the formula: (hospital reimbursements-hospital total costs), with the median hospital margin calculated from the dataset of the hospital margin associated with each patient. The IDX Hospital Data Systems (GE Medical Systems) was used to account for physician fees. Analysis was limited to physician charges and reimbursement and not physician costs. The physician reimbursement data was limited to surgeons' reimbursements. Total insurance liability was obtained by combining the TSI hospital reimbursement data and IDX physician reimbursement data via the formula: (hospital reimbursements + physician reimbursements). The median total insurer reimbursement was calculated from the dataset of the total insurer reimbursement associated with each patient.

\section{Statistical analysis}

The association of donor and transplant recipient variables was assessed using an unpaired Student's $t$-test for continuous variables and a chi square analysis for categorical variables. The donor age was unknown for three transplant recipients. Differences in financial data medians were assessed via the Mann-Whitney U-test for comparison of medians. The relationship between cold ischemia time and costs was assessed using a simple linear regression. Only the 35 patients for whom reliable cold ischemia data was available were included in this particular analysis $(n=22$ for patients with no surgical complication, $\mathrm{n}=13$ for patients with a surgical complication). Relationships were considered significant if $p \leq 0.05$. All statistical analyses were performed using Statview (version 5.0.1) (Abacus Concepts, Inc. Berkley, CA).

\section{Results}

The baseline characteristics of the study population (49 pancreas transplants done between 1/6/2002 and 11/22/2004) are detailed in Table 1. There were no significant differences in age between patients or donors for those who had a surgical complication versus those who did not have a surgical complication. Men made up a relatively higher percentage of those who did have a surgical complication, but this difference was not significant. Cold ischemia time was significantly shorter for those who had no surgical complication versus those who did (mean difference $2.9 \mathrm{~h}$, $p=0.002$ ). As expected, complications were associated with more hospital days immediately following the procedure (mean difference 5.7 days, $p=0.005)$ and a trend toward more readmissions in the first 6 months $(P=N S)$.

The financial data on the study cohorts (surgical complication and no surgical complication) are summarized in Table 2. Surgical complications were associated with significantly increased hospital charges, costs and reimbursements. Interestingly, the median hospital margin (estimated) was actually higher in patients with a surgical complication, though this difference, which totaled $\$ 11,369$, was not statistically significant. The median costs to the hospital were $\$ 15,514$ higher $(p=$ $0.03)$, and total estimated reimbursement by the payer, which includes surgeons' actual reimbursement in addition to estimated hospital reimbursements, was $\$ 17,363(p=0.001)$ higher in patients with a surgical complication

Hospital bottom lines were closely related to the type of procedure being done, i.e. solitary pancreas transplants (PTA, $n=2$ or PAK, $n=16$ ) or simultaneous pancreas-kidney (SPK, $n=31$ ), as detailed in Table 3. There 


\section{Cohn et al.}

Table 2: Financial data of patients with no surgical complication vs. those with a surgical complication

\begin{tabular}{|c|c|c|c|c|c|c|}
\hline \multirow[b]{2}{*}{ Dollars (\$) } & \multicolumn{2}{|c|}{$\begin{array}{l}\text { No surgical } \\
\text { complication } \\
(\mathrm{n}=30)\end{array}$} & \multicolumn{2}{|c|}{$\begin{array}{l}\text { Surgical } \\
\text { complication } \\
(n=19)\end{array}$} & \multirow{2}{*}{$\begin{array}{l}\text { Difference } \\
\text { in median }\end{array}$} & \multirow[b]{2}{*}{ p-Value } \\
\hline & Median & IQR & Median & IQR & & \\
\hline Hospital charges & 122,704 & 35,511 & 155,859 & 66,269 & $-33,155$ & 0.006 \\
\hline Total cost to hospital & 92,917 & 43,313 & 108,431 & 45,094 & $-15,514$ & 0.03 \\
\hline Estimated hospital reimbursement & 91,429 & 17,896 & 107,148 & 43,768 & $-15,719$ & 0.001 \\
\hline Estimated hospital margin (profit) & $-4,692$ & 28,075 & 6,677 & 65,919 & $-11,639$ & 0.21 \\
\hline Reimbursement for physician services & 2,512 & 1,252 & 2,921 & 2,925 & -409 & 0.14 \\
\hline Estimated total insurance liability & 93,497 & 19,915 & 110,860 & 46,079 & $-17,363$ & 0.001 \\
\hline
\end{tabular}

were no significant differences between these two groups in terms of age at transplant, gender, donor age, cold ischemia time, readmission, length of stay or frequency of complications (Table 4). While SPK procedures were significantly more costly for the medical center, there was no significant difference in reimbursement when compared to solitary pancreas transplants. As such, the difference in median margin was substantial, with UMHS making an estimated profit of $\$ 34,022$ on pancreas transplant-only procedures versus taking a loss of $\$ 10,058$ on SPK procedures, a difference of $\$ 44,080$ $(p<0.001)$.

In order to control for variation in data specifically related to payer and procedure mix, we compared patients with and without surgical complications that underwent an SPK transplant and had Medicare insurance. (Table 5) There were significantly higher medical center costs and insurer costs among the patients with surgical complications in this group of patients. The estimated hospital margin was higher in the group of patients with complications, thought this difference was not statistically significantly. A similar analysis was completed comparing the financial implications of complications among PAK patients with Medicare (Table 6). There were also higher medical center costs and insurer costs, with lower medical center margins in the group of patients with surgical complications. These differences were not significant in this small sample. Finally, in patients with complications, higher costs of the medical center and insurer were noted in SPK patients with private insurance and PAK patients with private insurance (data not shown).

\section{Discussion}

In order to build the case for transplant surgical quality improvement as a worthwhile investment, it is important to assign costs to items designated for improvement.
Troppman et al. studied the rates of complications associated with pancreas transplantation but noted the need for a detailed financial analysis (5). To this end, we analyzed surgical complications following pancreas transplantation to build a financial argument for quality improvement. The results suggest that payers should take a particular interest in transplant quality improvement, since they bear the biggest financial burden of surgical complications following pancreas transplantation.

Surgical complications following pancreas transplantation increase costs for the medical center and for the payer. Specifically, a pancreas transplant with a surgical complication increased hospital costs by $\$ 15,514$, while payers reimbursed UMHS an additional \$17,363 per case. Interestingly, this implies the medical center makes more profit when there is a surgical complication associated with a pancreas transplant. These findings underscore the important impact of surgical complications on medical center finances.

It must be noted, however, that hospital margin correlated more with the type of procedure (i.e. simultaneous pancreas-kidney or pancreas alone) than with whether there was a surgical complication, and rather substantially at that. The difference in margin was primarily a consequence of similar (and counterintuitive) reimbursements for solitary pancreas transplants and simultaneous kidneypancreas transplants, despite significantly higher costs for the combined procedure. This illustrates that costs are

Table 3: Financial implications of the type of transplant procedure

\begin{tabular}{|c|c|c|c|c|c|c|}
\hline \multirow[b]{2}{*}{ Dollars (\$) } & \multicolumn{2}{|c|}{$\begin{array}{l}\text { Simultaneous } \\
\text { pancreas-kidney } \\
(n=31)\end{array}$} & \multicolumn{2}{|c|}{$\begin{array}{l}\text { Solitary } \\
\text { pancreas transplant } \\
\text { (PTA or PAK) }(n=18)\end{array}$} & \multirow{2}{*}{$\begin{array}{l}\text { Difference } \\
\text { in median }\end{array}$} & \multirow[b]{2}{*}{ p-Value } \\
\hline & Median & IQR & Median & IOR & & \\
\hline Hospital charges & 134,777 & 43,578 & 95,181 & 61,344 & 39,596 & 0.002 \\
\hline Total cost to hospital & 107,298 & 26,115 & 57,728 & 41,722 & 49,570 & $<0.001$ \\
\hline Estimated hospital reimbursement & 96,788 & 25,489 & 98,063 & 49,323 & -1275 & 0.62 \\
\hline Estimated hospital margin (profit) & $-10,058$ & 26,059 & 34,022 & 43,297 & $-44,080$ & $<0.001$ \\
\hline Reimbursement for physician services & 2765 & 2082 & 2772 & 2658 & -7 & 0.76 \\
\hline Estimated total insurance liability & 101,402 & 24,348 & 100,969 & 48,972 & 433 & 0.58 \\
\hline
\end{tabular}


Cost of Pancreatic Transplant Complications

Table 4: Background data of SPK and solitary pancreas transplant recipients (data reported as mean \pm SD)

\begin{tabular}{|c|c|c|c|c|}
\hline $\begin{array}{l}\text { Type of } \\
\text { procedure }\end{array}$ & $\begin{array}{l}\text { Simultaneous } \\
\text { pancreas-kidney } \\
(\mathrm{n}=31)\end{array}$ & $\begin{array}{l}\text { Solitary pancreas } \\
\text { transplant } \\
\text { (PTA or PAK) }(n=18)\end{array}$ & $\begin{array}{l}\text { Mean } \\
\text { difference }\end{array}$ & $\mathrm{p}$-Value \\
\hline Recipient age (years) & $38.6 \pm 5.9$ & $38.2 \pm 5.7$ & 0.4 & 0.80 \\
\hline Donor age (years) & $26.5 \pm 10.8$ & $22.8 \pm 9.5$ & 3.7 & 0.24 \\
\hline Cold ischemia time (hours) & $9.0 \pm 2.5$ & $8.7 \pm 3.5$ & 0.3 & 0.82 \\
\hline Readmissions first 6 months & $0.9 \pm 1.1$ & $1.5 \pm 1.3$ & 0.6 & 0.11 \\
\hline Length of stay (days) & $7.9 \pm 7.1$ & $8.2 \pm 7.5$ & 0.3 & 0.91 \\
\hline Surgical complication rate & $10(32 \%)$ & $9(50 \%)$ & - & 0.22 \\
\hline
\end{tabular}

but one part of the picture when analyzing the potential impact of quality improvement initiatives, and that these analyses must be interpreted in light of the corresponding reimbursements. This counterintuitive reimbursement for solitary pancreas transplants has been noted and addressed by CMS. Specifically, the CMS reimbursement for solitary pancreas transplant has been reduced by $-31.6 \%$ for 2007. This reimbursement change will likely be followed by private payers and will significantly reduce the high margins for solitary pancreas transplant enjoyed by our center.

It should be noted, however, that any financial data is highly institution specific and use of our financial data for contract negotiation would not be appropriate. There are significant differences in cost-accounting methods between medical centers and even within our center from a yearto-year basis. Additional differences between institutions include personnel salaries, cost of living adjustments, contract rates of payment per organ and indirect costs (i.e. hospitals in the city are more expensive to operate). In an attempt to make the analysis more generalizable, we analyzed just Medicare patients who underwent an SPK transplant as well as other subsets of payer and procedure mix not. The general theme that insurers bear the costs of surgical complications was consistent through all of these analyses and is likely applicable to a broad range of centers, but the specific financial data should be assessed with caution.

For multiple reasons, our study is limited in scope. First, it fails to quantify opportunity costs for the medical center, which runs at capacity. For example, having a pancreas transplant patient in a bed prevents another, potentially 'profitable' patient from occupying that bed. Thus, the perceived increase in cost from complications may be higher for the medical center and the profit lower. Second, it focuses on the financial implications to the payer, the medical center, and the providers, but not costs to the patient or society. Certainly a complicated post pancreas transplant course will deleteriously affect future patient productivity. This may be even more significant than for other organ transplant recipients given the relatively young age of pancreas transplant recipients. Third, the cost data is based on the UMHS cost accounting methods, and accounting assumptions are inherently imperfect and will have significant impact on data and associated conclusions. Fourth, the physician reimbursement data includes only surgeons' reimbursement. It fails to account for reimbursements to all other physicians involved in patient care, which may be even more significant for patients who have suffered a surgical complication. Similarly, the costs are limited to those occurring within UMHS (though we estimate that most of our patients return to UMHS for all of their care in the posttransplant period), thus costs and reimbursements are likely disproportionately underestimated for patients with complications. Thus, the total insurer reimbursement is an underestimate of actual payer costs. In addition, because pancreas transplantation is relatively uncommon compared with other abdominal organ transplants, our patient sample size is limited. Reported complication rates following pancreas transplantation vary widely. Our overall complication rate $(38 \%)$ is higher end of this range $(3)$, thus the results may not be completely applicable to other centers.

Table 5: Medicare SPK patients only. Financial data of patients with no surgical complication vs. those with a surgical complication among patients undergoing an SPK transplant who had Medicare insurance

\begin{tabular}{|c|c|c|c|c|c|c|}
\hline \multirow[b]{2}{*}{ Dollars (\$) } & \multicolumn{2}{|c|}{$\begin{array}{l}\text { No surgical } \\
\text { complication } \\
(n=16)\end{array}$} & \multicolumn{2}{|c|}{$\begin{array}{l}\text { Surgical } \\
\text { complication } \\
(n=7)\end{array}$} & \multirow{2}{*}{$\begin{array}{l}\text { Difference } \\
\text { in median }\end{array}$} & \multirow[b]{2}{*}{$\mathrm{p}$-Value } \\
\hline & Median & IQR & Median & IQR & & \\
\hline Total cost to hospital & 100,350 & 17,508 & 125,457 & 60,686 & 25,107 & 0.004 \\
\hline Estimated hospital reimbursement & 91,429 & 13,521 & 108,818 & 30,735 & 17,389 & 0.003 \\
\hline Estimated hospital margin (profit) & $(12,615)$ & 22,037 & (9405) & 22,275 & 3210 & 0.55 \\
\hline Reimbursement for physician services & 2815 & 1899 & 2920 & 2425 & 105 & 0.88 \\
\hline Estimated total insurance liability & 93,496 & 13,360 & 110,859 & 31,171 & 17,353 & 0.002 \\
\hline
\end{tabular}




\section{Cohn et al.}

Table 6: Medicare PAK patients only. Financial data of patients with no surgical complication vs. those with a surgical complication among patients undergoing a PAK transplant who had Medicare insurance

\begin{tabular}{|c|c|c|c|c|c|c|}
\hline \multirow[b]{2}{*}{ Dollars (\$) } & \multicolumn{2}{|c|}{$\begin{array}{l}\text { No surgical } \\
\text { complication } \\
(n=6)\end{array}$} & \multicolumn{2}{|c|}{$\begin{array}{l}\text { Surgical } \\
\text { complication } \\
(\mathrm{n}=4)\end{array}$} & \multirow{2}{*}{$\begin{array}{l}\text { Difference } \\
\text { in median }\end{array}$} & \multirow[b]{2}{*}{ p-Value } \\
\hline & Median & IQR & Median & IQR & & \\
\hline Total cost to hospital & 56,938 & 20,886 & 74,491 & 49,919 & 17,553 & 0.28 \\
\hline Estimated hospital reimbursement & 94,988 & 23,750 & 106,735 & 32,455 & 11,747 & 0.20 \\
\hline Estimated hospital margin (profit) & 52,963 & 25,368 & 38,072 & 23,491 & 14,891 & 0.28 \\
\hline Reimbursement for physician services & 2505 & 951 & 2513 & 1210 & 8 & 0.98 \\
\hline Estimated total insurance liability & 108,485 & 32,762 & 97,016 & 24,177 & 11,469 & 0.20 \\
\hline
\end{tabular}

We have identified general trends, but a larger cohort is needed to verify the accuracy of the calculated cost figures.

Though our complication rate is higher than other reported series and therefore not necessarily generalizable, if we applied the surgical complication rate of $38 \%$ associated with pancreas transplants obtained from our study population to all pancreas transplants performed in the United States during the year 2004 ( $n=1484)$ (6), there were approximately 564 pancreas transplants with surgical complications across the country. Using our data, hospitals spent an additional $\$ 8.8$ million, and insurance carriers reimbursed medical centers an additional $\$ 9.8$ million as a result of surgical complications associated with pancreas transplantation. Bearing in mind that many complications may not be preventable, achieving the reasonable goal of a twenty percent reduction in the rate of pancreas transplant surgical complications could lead to a savings of nearly $\$ 2,000,000$ dollars per year for insurers. Based on this logic, insurer funding of a national quality improvement initiative may be a reasonable investment.

In summary, pancreas transplantation, with a wide variety of surgical approaches and high complication rates, represents an operation particularly well suited to multicenter quality improvement initiatives. A national quality improvement program would provide an infrastructure to identify significant outliers for high quality, and subsequently report those centers' techniques and protocols in the literature. Self-reporting of complication rates is inherently inadequate to determine who has the best pancreas transplant results and simply focusing on graft survival does not provide sufficient insight. These outcomes must be collected prospectively by highly trained personnel, be based on uniform definitions, and the data collection accuracy must be audited. This effort would create a culture of quality improvement and allow programs with inferior results to re- view the specific approaches of the high quality centers. This infrastructure will be expensive; therefore we suggest that a transplant quality improvement may be a sound investment for payers.

\section{Acknowledgments}

MJE is supported by an American Surgical Foundation Grant. DAC is supported by a grant from the Blue Cross and Blue Shield Foundation.

\section{References}

1. Khuri SF, Daley J, Henderson W et al. The Department of Veterans Affairs' NSOIP: The first national, validated, outcome-based, riskadjusted, and peer-controlled program for the measurement and enhancement of the quality of surgical care. Ann Surg 1998; 228: 491-507.

2. Englesbe Michael J., Pelletier SJ, Kheterpal S, O'Reilly M, Campbell DAJr. A call for a national transplant surgical quality improvement program. Am J Transplant 2006; 6: 666-670.

3. Gruessner AC, Troppmann C, Sutherland DE, Gruessner RW. Donor and recipient risk factors significantly affect cost of pancreas transplants. Transplant Proc 1997; 29: 656-657.

4. Pronovost $P$, Angus DC. Cost reduction and quality improvement: It takes two to tango. Crit Care Med 2000; 28: 581-583.

5. Troppmann C, Gruessner AC, Dunn DL, Sutherland DE, Gruessner RW. Surgical complications requiring early relaparotomy after pancreas transplantation: A multivariate risk factor and economic impact analysis of the cyclosporine era. Ann Surg 1998; 227: 255268.

6. 2005 Annual Report of the U.S. Organ Procurement and Transplantation Network and the Scientific Registry for Transplant Recipients: Transplant Data 1995-2004: Department of Health and Human Services, Health Resources and Services Administration, Office of Special Programs, Division of Transplantation, Rockville, MD; United Network for Organ Sharing, Richmond, VA; University Renal Research and Education Association, Ann Arbor, MI, 2005. 